\title{
Estimation of the Scale parameter from the Rayleigh distribution from type II singly and doubly censored data
}

\author{
Ahmad Saeed Akhter \\ College of Statistical \& Actuarial Sciences \\ University of the Punjab \\ Q.A. Campus, Lahore \\ Abdul Samad Hirai \\ 52 Mamdot Block \\ Mustafa Town, Lahore
}

\section{Introduction}

As common as the normal distribution is the Rayleigh distribution which occurs in works on radar, properties of sine wave plus-noise, etc. Rayleigh (1880) derived it from the amplitude of sound resulting from many important sources. The Rayleigh distribution is widely used in communication engineering, reliability analysis and applied statistics. Since the Rayleigh distribution has linearly increasing rate, it is appropriate for components which might not have manufacturing defects but age rapidly with time. Several types of electro-vacum devices have this feature. It is connected with one dimension and two dimensions random walk and is some times referred to as a random walk frequency distribution. It is a special case of Weibull distribution (1951) of wide applicability. It can be easily derived from the bivariate normal distribution with $\sigma_{1}=\sigma_{2}=\sigma$ and $p=0$. For further application of Rayleigh distribution, we refer to Johnson and Kotz (1994). Adatia (1995) has obtained the best linear unbiased estimator of the Rayleigh scale parameter based on fairly large censored samples. Dyer and Whisend (1973) obtained the BLUE of scale parameter based on type II censored samples for small $N=2(1) 5$. With the advance of computer technology it is now possible to obtain BLUE for large samples. Hirai (1978) obtained the estimate of the scale parameter from the Rayleigh distribution singly type II censored from the left side and right side and variances of the scale parameter. In this paper, we estimate the scale parameter of type II singly and doubly censored data from the Rayleigh distribution using Blom's (1958) unbiased nearly best estimates and compare the efficiency of this estimate with BLUE and MLE.

\section{The Rayleigh Distribution}

The probability density function of the p-dimensional Rayleigh distribution is

$$
\begin{aligned}
f(Z, p, \lambda) & =\frac{2^{-(p-2)}}{\lambda \Gamma\left(\frac{p}{2}\right)}\left(\frac{Z}{\lambda}\right)^{p-1} \exp \left[-\frac{1}{2}\left(\frac{Z}{\lambda}\right)\right]^{p} \quad Z \geq 0 \\
& =0 \quad \text { otherwise }
\end{aligned}
$$


The general form for $p=3$ has limited applications. We consider the special case when $\mathrm{p}=2$ and $\mathrm{Z}=\sqrt{2} x$ warrants sufficient interest from the application point of view. Now the p.d.f. of the standard Rayleigh distribution is

$$
\begin{array}{rl}
f(x)=\frac{2 x}{\lambda^{2}} e^{-x^{2} / \lambda^{2}} & x>0 \\
\lambda & >0
\end{array}
$$

with $\quad P(X \leq x)=F(x)=1-e^{-x^{2} / \lambda^{2}}$

\section{Moments of order statistics from the Rayleigh distribution}

The c.d.f. of the Rayleigh distribution is

$$
F(x)=1-e^{-x^{2} / \lambda^{2}}
$$

If $x_{(1)}^{(n)}<x_{(2)}^{(n)}<\ldots \ldots . .<x_{(r)}^{(n)} \ldots \ldots . .<x_{(n)}^{(n)}$ are the available ordered observation from

(1). Let us make the transformation $\frac{y_{(r)}^{(n)}-x_{(r)}^{(n)}}{\lambda}$ then the observations $y_{(1)}^{(n)}<y_{(2)}^{(n)}<\ldots \ldots . .<y_{(n)}^{(n)}$ are the realizations of random variables $Y_{(1)}^{(n)}<Y_{(2)}^{(n)}<\ldots \ldots . .<Y_{(n)}^{(n)}$.

Let $E\left(Y_{(r)}^{(n)}\right)=\alpha_{(r)}^{(n)} ; E\left(Y_{(r)}^{(n)}, Y_{(r)}^{(n)}\right)=W_{r s}^{(n)}$

and $\operatorname{Cov}\left(Y_{(r)}^{(n)}, Y_{(s)}^{(n)}\right)=W_{r s}^{(n)}-\alpha_{r}^{(n)} \alpha_{s}^{(n)}=V_{r s}^{(n)} r<s$

The derivation of $\alpha_{r}^{(n)}$ from the Rayleigh distribution is straight forward and we have

$$
\alpha_{r}^{(n)}=\frac{n ! \sqrt{\pi}}{2(n-r) !(r-1) !} \sum_{i=0}^{r-1}\left(\begin{array}{c}
i-1 \\
i
\end{array}\right)(-1)^{i} \frac{1}{(i+n-r+1)^{3 / 2}}
$$

The evaluation of the exact variance - covariance matrix of the Rayleigh distribution is somewhat complicated. Hirai (1976) evaluated the expected vector and $V_{r S}^{(n)}$ for $n \leq 8$ and Govindarajulu, $Z$ and Goshi (1968) give values for sample sizes $2-10$.

4. Maximum likelihood estimate of the scale parameter $\lambda$ from the Rayleigh distribution

We have the p.d.f. of the Rayleigh distribution

$$
f(x)=\frac{2 x}{\lambda^{2}} e^{-x^{2} / \lambda^{2}} \quad 0 \leq x \leq \infty
$$


The likelihood function of a sample size $\mathrm{n}$ from this distribution is

$$
L=\frac{2^{n}}{\lambda^{2 n}} \sum_{i=1}^{n} x_{i} e^{-\sum_{i=1}^{n} x_{i}^{2} / \lambda^{2}}
$$

and from this we obtain M.L.E. $\hat{\lambda}$ of $\lambda$ as

$$
\hat{\lambda}=\left[\frac{1}{n} \sum x_{i}^{2}\right]^{1 / 2} \text {; thus } \frac{\hat{\lambda} \sqrt{2 n}}{\lambda} \text { is a } \chi \text { variate with } 2 \mathrm{n} \text { d.f. }
$$

and $\quad d F(\hat{\lambda})=\frac{2(\sqrt{n})^{2 n}}{\lambda^{2 n} \Gamma(n)} e^{\frac{-n \hat{\lambda}^{2}}{\hat{\lambda}^{2}}}(\hat{\lambda})^{2 n-1} d \lambda 0<\hat{\lambda}<\infty$

and $\quad \mu_{1}^{\prime}(\hat{\lambda})=\frac{\Gamma \frac{2 n+1}{2}}{\Gamma(n)} \frac{\lambda}{\sqrt{\Pi}} \quad$ and $\quad \mu_{2}^{\prime}(\hat{\lambda})=\lambda^{2}$

It can be easily shown that the distribution of $\hat{\lambda}$ tends to normality as $\mathrm{n}$ becomes very large.

Also we have $\quad \operatorname{Var}(\hat{\lambda})=\frac{\lambda^{2}}{4 n} \quad$ (Cramer Rao lower bound)

Hirai (1976) has obtained the variance of $\hat{\lambda}$ for $n \leq 8$ for various values of $r_{2}$ which is fixed in advance (right tail censored) using M.L.E.

Where $x_{\left(r_{2}\right)}^{(n)}$ is the largest observation available out of sample size $\mathrm{n}$ from the Rayleigh distribution in table 1.

Table 1: Showing the variance of $\hat{\lambda}$ for different values of $r_{2}$. Each value should be multiplied by $\lambda^{2}$

\begin{tabular}{|c|c|c|c|c|c|c|c|}
\hline$r_{2}$ & 1 & 2 & 3 & 4 & 5 & 6 & 7 \\
\hline$n$ & 2 & 3 & 4 & 5 & 6 & 7 & 8 \\
\hline $\operatorname{Var}(\hat{\lambda})$ & 0.2500000 & 0.1250000 & 0.0533333 & 0.0625000 & 0.0500000 & 0.041667 & 0.0357143 \\
\hline
\end{tabular}

Similarly when $r_{1}$ left side censored are missing observations out of sample size 8 and variance of $\hat{\lambda}$ are shown in table 2 using M.L.E. 
Table 2: Showing the variance of $\hat{\lambda}$ for $n \leq 8$ for various values of $\mathbf{r}_{1}$. Each value may be multiplied by $\lambda^{2}$

\begin{tabular}{|c|c|c|c|c|c|c|c|}
\hline$r_{1}$ & $\mathbf{1}$ & $\mathbf{2}$ & $\mathbf{3}$ & $\mathbf{4}$ & $\mathbf{5}$ & $\mathbf{6}$ & $\mathbf{7}$ \\
\hline $\mathrm{n}=2$ & 0.1428 & & & & & & \\
\hline $\mathrm{n}=3$ & 0.0861 & 0.1111 & & & & & \\
\hline $\mathrm{n}=4$ & 0.0635 & 0.0784 & 0.0952 & & & & \\
\hline $\mathrm{n}=5$ & 0.0505 & 0.0518 & 0.0557 & 0.0787 & & & \\
\hline $\mathrm{n}=6$ & 0.0420 & 0.0425 & 0.0439 & 0.0482 & 0.0787 & & \\
\hline $\mathrm{n}=7$ & 0.0359 & 0.0362 & 0.0368 & 0.0384 & 0.0429 & 0.0738 & \\
\hline $\mathrm{n}=8$ & 0.0314 & 0.0316 & 0.0319 & 0.0326 & 0.0343 & 0.0389 & 0.0699 \\
\hline
\end{tabular}

\section{Estimation of $\lambda$ by BLUE}

Lloyd (1952) considered the estimation of location and scale parameters using ordered statistics for the continuous distributions with c.d.f. $=F\left(\frac{x-\mu}{\lambda}\right)$. The method can easily be extended to a potential sample of size $n$, a known number of observations is missing at either end (single censoring) or at both ends (doubly censoring) i.e. Type II censoring. (Gupta 1952) to distinguish it from the situation in which the sample is curtailed below and / or above a fixed point. Adatia (1995) has obtained the coefficients and relative s-efficiency of the best linear unbiased estimate (BLUE) of the scale parameter of the Rayleigh distribution for type II censored samples of size $N=20$ (5) 40 for $r=0$ (1) 4 number of observations censored from the left and $s=0$ (1) 4 (number of observations censored from the right). Dyer and Whisenand (1973) obtained the BLUE of the scale parameter based on type II censored samples for small $n=2$ (1) 15 .

6. Best Linear Unbiased estimation of $\lambda$, the scale parameter of the Rayleigh distribution by Lloyds method for type II censored data

Suppose that $x_{(r+1)}^{(n)}<x_{(r+2)}^{(n)} \leq \ldots \ldots . . \leq x_{(n-s)}^{(n)}$ are the available type II censored sample, where the smallest $r$ and the largest $s$ observations were not observed due to experimental restrictions. Then using Lloyd's method $\lambda^{*}$ an estimate of $\lambda$ is given as:

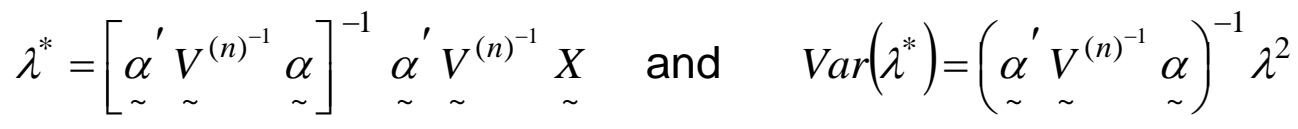


where

$$
\underset{\sim}{X}=\left[\begin{array}{c}
x_{(r+1)}^{(n)} \\
x_{(r+2)}^{(n)} \\
\mathrm{M} \\
x_{(n-r-s+1)}^{(n)}
\end{array}\right] \text { and } \quad \underset{\sim}{\alpha}=\left[\begin{array}{lll}
\alpha_{(r+1)}^{(n)} & \mathrm{K} & \alpha_{(n-r-s+1)}^{(n)}
\end{array}\right]
$$

and $V^{(n)}$ is the exact sub variance covariance matrix

$$
\stackrel{\sim}{V^{(n)}}=\left[\begin{array}{cccc}
V_{r+1, r+1}^{(n)} & V_{r+1, r+2}^{(n)} & \mathrm{K} & V_{r+1, n-r-s}^{(n)} \\
V_{r+2, r+1}^{(n)} & V_{r+2, r+2}^{(n)} & \mathrm{K} & V_{r+2, n-r-s}^{(n)} \\
V_{n-r-s+1, r+1}^{(n)} & \mathrm{K} & \mathrm{K} & V_{n-r-s, n-r-s+1}^{(n)}
\end{array}\right]
$$

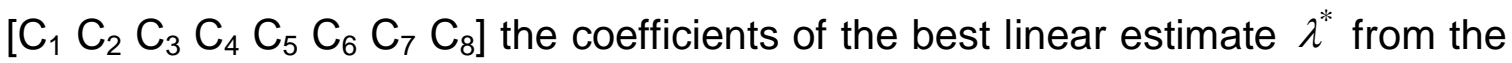
singly and doubly censored samples are given in table 3 and table 4 shows the exact variances of the estimate $\lambda^{*}$ from the Rayleigh distribution from singly and doubly censored samples.

Table 3: Showing the coefficients of the best linear estimate of $\lambda^{*}$ from Rayleigh distribution from singly and doubly censored samples

\begin{tabular}{|c|c|c|c|c|c|c|c|c|c|c|}
\hline $\mathbf{n}$ & $\mathbf{r}_{1}$ & $\mathbf{r}_{2}$ & $\mathbf{C}_{1}$ & $\mathbf{C}_{2}$ & $\mathbf{C}_{3}$ & $\mathbf{C}_{4}$ & $\mathbf{C}_{5}$ & $\mathbf{C}_{6}$ & $\mathbf{C}_{7}$ & $\mathbf{C}_{8}$ \\
\hline 3 & 0 & 1 & 0.2712550 & 1.0053272 & & & & & & \\
\hline & 1 & 0 & & 0.3989254 & 0.5108640 & & & & \\
& 0 & 1 & 0.1580963 & 0.2465317 & 0.7561479 & & & & & \\
& 0 & 2 & 0.2322999 & 1.2505903 & & & & & \\
& 1 & 0 & & 0.2534338 & 0.2612984 & 0.4018431 & & & \\
& 1 & 1 & & 0.1799068 & 0.6667720 & & & & \\
& 2 & 0 & & & 0.4228779 & 0.4168678 & & & \\
& 0 & 1 & 0.1064325 & 0.1631825 & 0.2167412 & 0.6131440 & & & \\
& 0 & 2 & 0.1407905 & 0.2142904 & 0.9542631 & & & & \\
& 0 & 3 & 0.2064764 & 1.4568612 & & & & & \\
& 1 & 0 & & 0.1802804 & 0.1759284 & 0.2276212 & 0.3338221 & & & \\
& 1 & 1 & & 0.2245908 & 0.2174143 & 0.6158018 & & & & \\
& 1 & 2 & & 0.2959298 & 0.9593902 & & & & & \\
\end{tabular}









\begin{tabular}{|c|c|c|c|c|c|c|c|c|c|c|}
\hline $\mathbf{n}$ & $\mathbf{r}_{1}$ & $r_{2}$ & $\mathbf{C}_{1}$ & $\mathrm{C}_{2}$ & $\mathrm{C}_{3}$ & $\mathrm{C}_{4}$ & $\mathrm{C}_{5}$ & $\mathrm{C}_{6}$ & $\mathrm{C}_{7}$ & $\mathrm{C}_{8}$ \\
\hline & 2 & 3 & & & 0.3055790 & 0.9372246 & & & & \\
\hline & 3 & 0 & & & & 0.2574127 & 0.1494079 & 0.1853637 & 0.2566061 & \\
\hline & 3 & 1 & & & & 0.3000117 & 0.1755594 & 0.4630005 & & \\
\hline & 3 & 2 & & & & 0.3588506 & 0.6851297 & & & \\
\hline & 4 & 0 & & & & & 0.3422117 & 0.1912039 & 0.2654203 & \\
\hline & 4 & 1 & & & & & 0.4005271 & 0.4810243 & & \\
\hline & 5 & 0 & & & & & & 0.4456277 & 0.2876659 & \\
\hline 8 & 0 & 1 & 0.0480569 & 0.0728980 & 0.0922028 & 0.1124096 & 0.1328922 & 0.1565398 & 0.4006700 & \\
\hline & 0 & 2 & 0.0550656 & 0.0872158 & 0.1058141 & 0.1305451 & 0.1535812 & 0.5860067 & & \\
\hline & 0 & 3 & 0.0675305 & 0.1002288 & 0.1284742 & 0.1550206 & 0.7960510 & & & \\
\hline & 0 & 4 & 0.0839051 & 0.1243542 & 0.1586005 & 1.0537224 & & & & \\
\hline & 0 & 5 & 0.1105934 & 0.1632030 & 1.4015645 & & & & & \\
\hline & 0 & 6 & 0.1617772 & 0.1067282 & & & & & & \\
\hline & 1 & 0 & & 0.0895308 & 0.0807210 & 0.0987684 & 0.1167561 & 0.1384433 & 0.1644683 & 0.2249580 \\
\hline & 1 & 1 & & 0.1022131 & 0.0922049 & 0.1124778 & 0.1329270 & 0.1567995 & 0.4010073 & \\
\hline & 1 & 2 & & 0.1208241 & 0.1058296 & 0.1306406 & 0.1536409 & 0.5867184 & & \\
\hline & 1 & 3 & & 0.1414755 & 0.1285269 & 0.1551742 & 0.7970814 & & & \\
\hline & 1 & 4 & & 0.1756574 & 0.1587143 & 1.0555599 & & & & \\
\hline & 1 & 5 & & 0.2309378 & 1.4042631 & & & & & \\
\hline & 2 & 0 & & & 0.1452545 & 0.0989330 & 0.1172864 & 0.1384930 & 0.1651467 & 0.2257141 \\
\hline & 2 & 1 & & & 0.1659589 & 0.1127183 & 0.1335947 & 0.1569278 & 0.4026902 & \\
\hline & 2 & 2 & & & 0.1931472 & 0.1310155 & 0.1545337 & 0.5890050 & & \\
\hline & 2 & 3 & & & 0.2309405 & 0.1557252 & 0.8010645 & & & \\
\hline & 2 & 4 & & & 0.2862135 & 1.0616379 & & & & \\
\hline & 3 & 0 & & & & 0.2092289 & 0.1180182 & 0.1395811 & 0.1664954 & 0.2276706 \\
\hline & 3 & 1 & & & & 0.2390290 & 0.1345940 & 0.1583557 & 0.4065896 & \\
\hline & 3 & 2 & & & & 0.2784587 & 0.1559346 & 0.5955457 & & \\
\hline & 3 & 3 & & & & 0.3327320 & 0.8113475 & & & \\
\hline & 4 & 0 & & & & & 0.2794656 & 0.1418949 & 0.1695684 & 0.2320723 \\
\hline & 4 & 1 & & & & & 0.3199120 & 0.1614220 & 0.4154270 & \\
\hline
\end{tabular}




\begin{tabular}{|c|c|c|c|c|c|c|c|c|c|c|}
\hline$n$ & $\mathbf{r}_{1}$ & $\mathbf{r}_{2}$ & $C_{1}$ & $\mathrm{C}_{2}$ & $\mathrm{C}_{3}$ & $\mathrm{C}_{4}$ & $\mathrm{C}_{5}$ & $\mathbf{C}_{6}$ & $C_{7}$ & $\mathbf{C}_{8}$ \\
\hline & 4 & 2 & & & & & 0.3731422 & 0.6102415 & & \\
\hline & 5 & 0 & & & & & & 0.3569806 & 0.1761485 & 0.2417729 \\
\hline & 5 & 1 & & & & & & 0.4100757 & 0.4348418 & \\
\hline & 6 & 0 & & & & & & & 0.4472739 & 0.2649313 \\
\hline
\end{tabular}

Table 4: Showing the exact variances of the estimate of $\lambda^{*}$ from the Rayleigh Distribution from singly and doubly censored samples.

Each value should be multiplied by $\lambda^{2}$.

\begin{tabular}{|c|c|c|c|c|c|c|c|c|}
\hline $\mathrm{n}$ & $r_{1}^{r_{2}}$ & 0 & 1 & 2 & 3 & 4 & 5 & 6 \\
\hline 3 & $\begin{array}{l}0 \\
1\end{array}$ & 0.0884688 & 0.1319092 & & & & & \\
\hline 4 & $\begin{array}{l}0 \\
1 \\
2\end{array}$ & $\begin{array}{l}0.0650335 \\
0.0676473\end{array}$ & $\begin{array}{l}0.0866286 \\
0.0874873\end{array}$ & 0.1318417 & & & & \\
\hline 5 & $\begin{array}{l}0 \\
1 \\
2 \\
3\end{array}$ & $\begin{array}{l}0.0515444 \\
0.0524295 \\
0.0555120\end{array}$ & $\begin{array}{l}0.0644327 \\
0.0647300 \\
0.0661093\end{array}$ & $\begin{array}{l}0.0865724 \\
0.0870934\end{array}$ & 0.1318131 & & & \\
\hline 6 & $\begin{array}{l}0 \\
1 \\
2 \\
3 \\
4\end{array}$ & $\begin{array}{l}0.0427159 \\
0.0431012 \\
0.0442478 \\
0.0475584\end{array}$ & $\begin{array}{l}0.0514102 \\
0.0514029 \\
0.0519522 \\
0.0536080\end{array}$ & $\begin{array}{l}0.0643910 \\
0.0645910 \\
0.0654428\end{array}$ & $\begin{array}{l}0.0865469 \\
0.0868971\end{array}$ & 0.1317991 & & \\
\hline 7 & $\begin{array}{l}0 \\
1 \\
2 \\
3 \\
4\end{array}$ & $\begin{array}{l}0.0364809 \\
0.0366751 \\
0.0371991 \\
0.0385295\end{array}$ & $\begin{array}{l}0.0425658 \\
0.0426304 \\
0.0428942 \\
0.0436088 \\
0.0454263\end{array}$ & $\begin{array}{l}0.0512665 \\
0.0513593 \\
0.0517372 \\
0.0527654\end{array}$ & $\begin{array}{c}0.0643388 \\
0.0644825 \\
0.0640653\end{array}$ & $\begin{array}{l}0.0865522 \\
0.0868026\end{array}$ & 0.1317830 & \\
\hline
\end{tabular}




\begin{tabular}{|c|c|c|c|c|c|c|c|c|}
\hline $\mathrm{n}$ & $\begin{array}{l}r_{2} \\
r_{1} \\
\end{array}$ & 0 & 1 & 2 & 3 & 4 & 5 & 6 \\
\hline & 5 & 0.0419216 & & & & & & \\
\hline 8 & 0 & & 0.0363893 & 0.0425391 & 0.0512256 & 0.0643575 & 0.0865234 & 0.1317846 \\
\hline & 1 & 0.0318354 & 0.0364248 & 0.0425857 & 0.0512957 & 0.0644658 & 0.0867117 & \\
\hline & 2 & 0.0319443 & 0.0364668 & 0.0427843 & 0.0515681 & 0.0648863 & & \\
\hline & 3 & 0.0322254 & 0.0369343 & 0.0432828 & 0.0522824 & & & \\
\hline & 4 & 0.0328646 & 0.0377708 & 0.0444222 & & & & \\
\hline & 5 & 0.0342816 & 0.0396390 & & & & & \\
\hline & 6 & 0.0377318 & & & & & & \\
\hline
\end{tabular}

7. Nearly unbiased best estimation of $\lambda$ say $\lambda_{1}{ }^{*}$ the scale parameter of the Rayleigh distribution by Blom's method

A notable feature of Blom's method is that it can readily be adapted to censored samples. Suppose, as before $r$ smallest and $s$ largest observations are missing (which covers the most common situation) then we seek the coefficients gi's based upon the observations $Z_{(r+1)}^{(n)}<Z_{\left(r_{1}+2\right)}^{(n)}<\ldots \ldots . . \ldots . . Z_{n-S}$.

The gi's corresponding to the missing observations are zero. For detail study of the Blom's method (1958) we refer to contributions to order statistics by Sarhan and Greenberg pages 35 to 40 .

Table 5 gives the coefficients of the nearly unbiased estimate $\lambda_{1}^{*}$ (say) of $\lambda$ from singly and doubly censored samples and table 6 gives the variances of $\lambda_{1}^{*}$ from the Rayleigh distribution for singly and doubly censored data for Rayleigh distribution. The variance of $\lambda_{1}^{*}$ given by Blom is very crude and otherwise the method works excellently and we only need the expected vectors. In order to compare this method with BLUE we sue sub-matrix of the exact variancecovariance Hirai (1978) for different values of $r$ and $s$ for a sample size $n \leq 8$. 
Table 5: Showing the coefficients of the nearly unbiased estimate of $\lambda_{1}{ }^{*}$ from the Rayleigh distribution from singly and doubly censored samples.

\begin{tabular}{|c|c|c|c|c|c|c|c|c|c|c|}
\hline $\mathrm{n}$ & $r_{1}$ & $r_{2}$ & $\mathbf{g}_{1}$ & $\mathbf{g}_{2}$ & $\mathbf{g}_{3}$ & $\mathbf{g}_{4}$ & $\mathbf{g}_{5}$ & $g_{6}$ & $g_{7}$ & $\mathbf{g}_{8}$ \\
\hline 3 & 1 & 0 & 0.1725257 & $\begin{array}{l}1.0642982 \\
0.3096864\end{array}$ & 0.5693779 & & & & & \\
\hline 4 & $\begin{array}{l}0 \\
1 \\
1 \\
2\end{array}$ & $\begin{array}{l}1 \\
2 \\
0 \\
1 \\
0\end{array}$ & $\begin{array}{l}0.0818326 \\
0.1299451\end{array}$ & $\begin{array}{l}0.2266848 \\
1.3138172 \\
0.1933817 \\
0.2739308\end{array}$ & $\begin{array}{l}0.8043716 \\
0.2212821 \\
0.8067522 \\
0.3446754\end{array}$ & $\begin{array}{l}0.4615712 \\
0.4729637\end{array}$ & & & & \\
\hline 5 & $\begin{array}{l}0 \\
0 \\
0 \\
1 \\
1 \\
1 \\
2 \\
2 \\
3\end{array}$ & $\begin{array}{l}1 \\
2 \\
3 \\
0 \\
1 \\
2 \\
0 \\
1 \\
0\end{array}$ & $\begin{array}{l}0.0441368 \\
0.0610580 \\
0.0961589\end{array}$ & $\begin{array}{l}0.1488822 \\
0.2059608 \\
1.5262324 \\
0.1337628 \\
0.1753656 \\
0.2426815\end{array}$ & $\begin{array}{l}0.1985789 \\
0.997230 \\
0.1516057 \\
0.1987580 \\
0.9989682 \\
0.2414983 \\
0.3176230\end{array}$ & $\begin{array}{l}0.6580007 \\
0.1952372 \\
0.6585942 \\
0.1972413 \\
0.6674855 \\
0.3631943\end{array}$ & $\begin{array}{l}0.3922307 \\
0.3962568 \\
0.4120623\end{array}$ & & & \\
\hline 6 & $\begin{array}{l}0 \\
0 \\
0 \\
0 \\
1 \\
1 \\
1 \\
1 \\
2 \\
2 \\
2\end{array}$ & $\begin{array}{l}1 \\
2 \\
3 \\
4 \\
0 \\
1 \\
2 \\
3 \\
0 \\
1 \\
2\end{array}$ & $\begin{array}{l}0.0247333 \\
0.0317812 \\
0.0436466 \\
0.0684953\end{array}$ & $\begin{array}{l}0.1071924 \\
0.1377379 \\
0.1891614 \\
1.7139246 \\
0.0980452 \\
0.1224444 \\
0.1573491 \\
0.2161246\end{array}$ & $\begin{array}{l}0.1413603 \\
0.1816421 \\
1.1645406 \\
\\
0.1132248 \\
0.1414016 \\
0.1817103 \\
1.1651413 \\
0.1815742 \\
0.2270456 \\
0.2922947\end{array}$ & $\begin{array}{l}0.1411011 \\
0.1762159 \\
0.8203417 \\
0.1418143 \\
0.1773287 \\
0.08270107\end{array}$ & $\begin{array}{l}0.1756848 \\
0.5654721\end{array}$ & 0.3429085 & & \\
\hline
\end{tabular}









\begin{tabular}{|c|c|c|c|c|c|c|c|c|c|c|}
\hline $\mathbf{n}$ & $r_{1}$ & $r_{2}$ & $g_{1}$ & $g_{2}$ & $g_{3}$ & $g_{4}$ & $g_{5}$ & $g_{6}$ & $g_{7}$ & $g_{8}$ \\
\hline & 1 & 1 & & 0.0689856 & 0.0857429 & 0.1036204 & 0.1223827 & 0.1433661 & 0.4408356 & \\
\hline & 1 & 2 & & 0.0820349 & 0.1019621 & 0.1234592 & 0.1455327 & 0.6185139 & & \\
\hline & 1 & 3 & & 0.1000738 & 0.1243828 & 0.1506070 & 0.8257807 & & & \\
\hline & 1 & 4 & & 0.1273365 & 0.1582679 & 1.0861877 & & & & \\
\hline & 1 & 5 & & 0.1738996 & 1.4481635 & & & & & \\
\hline & 2 & 0 & & & 0.1154129 & 0.0882251 & 0.1039990 & 0.1218304 & 0.1453934 & 0.277049 \\
\hline & 2 & 1 & & & 0.1360732 & 0.1040185 & 0.1226161 & 0.1436395 & 0.4416764 & \\
\hline & 2 & 2 & & & 0.1975643 & 0.1510240 & 0.8280674 & & & \\
\hline & 2 & 4 & & & 0.2516757 & 1.0900178 & & & & \\
\hline & 3 & 0 & & & & 0.1761128 & 0.1046212 & 0.1225592 & 0.1462632 & 0.278707 \\
\hline & 3 & 1 & & & & 0.2078618 & 0.1234819 & 0.1446538 & 0.4447950 & \\
\hline & 3 & 2 & & & & 0.2476017 & 0.1470897 & 0.6851313 & & \\
\hline & 3 & 3 & & & & 0.3027599 & 0.8365837 & & & \\
\hline & 4 & 0 & & & & & 0.2405198 & 0.1243742 & 0.1484292 & 0.282834 \\
\hline & 4 & 1 & & & & & 0.2846396 & 0.1471843 & 0.4525901 & \\
\hline & 4 & 2 & & & & & 0.3401982 & 0.6382251 & & \\
\hline & 5 & 0 & & & & & & 0.3091231 & 0.1532781 & 0.292074 \\
\hline & 5 & 1 & & & & & & 0.3680528 & 0.4701931 & \\
\hline & 6 & 0 & & & & & & & 0.3863513 & 0.313487 \\
\hline
\end{tabular}

Table 6: Showing the variance of nearly best linear estimate of $\lambda_{1}{ }^{*}$ from the Rayleigh distribution from singly and doubly censored samples. Each value should be multiplied by $\lambda^{2}$.

\begin{tabular}{|c|l|l|c|c|c|c|c|c|}
\hline $\mathbf{n}$ & $\mathbf{r}_{2}$ & $\mathbf{0}$ & $\mathbf{1}$ & $\mathbf{2}$ & $\mathbf{3}$ & $\mathbf{4}$ & $\mathbf{5}$ & $\mathbf{6}$ \\
\hline 3 & 0 & & 0.1323950 & & & & & \\
& 1 & 0.0890871 & & & & & & \\
\hline 4 & 0 & & 0.0869890 & 0.1322026 & & & & \\
& 1 & 0.0656243 & 0.0876400 & & & & & \\
& 2 & 0.0680903 & & & & & & \\
\hline
\end{tabular}




\begin{tabular}{|c|c|c|c|c|c|c|c|c|}
\hline$n$ & $r_{1}^{r_{2}}$ & 0 & 1 & 2 & 3 & 4 & 5 & 6 \\
\hline 5 & $\begin{array}{l}0 \\
1 \\
2 \\
3\end{array}$ & $\begin{array}{l}0.0520555 \\
0.0528791 \\
0.0558633\end{array}$ & $\begin{array}{l}0.0647015 \\
0.0648921 \\
0.0662025\end{array}$ & $\begin{array}{l}0.0868216 \\
0.0871714\end{array}$ & 0.1321332 & & & \\
\hline 6 & $\begin{array}{l}0 \\
1 \\
2 \\
3 \\
4\end{array}$ & $\begin{array}{l}0.0431551 \\
0.0435044 \\
0.0446133 \\
0.0478502\end{array}$ & $\begin{array}{l}0.0514833 \\
0.0515496 \\
0.0520612 \\
0.0536776\end{array}$ & $\begin{array}{l}0.0643682 \\
0.0646757 \\
0.0654814\end{array}$ & $\begin{array}{l}0.0867575 \\
0.0869536\end{array}$ & 0.1321001 & & \\
\hline 7 & $\begin{array}{l}0 \\
1 \\
2 \\
3 \\
4\end{array}$ & $\begin{array}{l}0.0368563 \\
0.0370295 \\
0.0375360 \\
0.0388379 \\
0.0421731\end{array}$ & $\begin{array}{l}0.0427352 \\
0.0427579 \\
0.0429979 \\
0.0436936 \\
0.0454808\end{array}$ & $\begin{array}{l}0.0514034 \\
0.0514372 \\
0.0517866 \\
0.0527945\end{array}$ & $\begin{array}{l}0.0644848 \\
0.0645389 \\
0.0650856\end{array}$ & $\begin{array}{l}0.0867507 \\
0.0868495\end{array}$ & 0.1320943 & \\
\hline 8 & $\begin{array}{l}4 \\
5\end{array}$ & $\begin{array}{l}0.0321623 \\
0.0322564 \\
0.0325256 \\
0.0331526 \\
0.0345505 \\
0.0379509\end{array}$ & $\begin{array}{l}0.0365323 \\
0.0365391 \\
0.0366644 \\
0.0370182 \\
0.0378408 \\
0.0396874\end{array}$ & $\begin{array}{c}0.0426465 \\
0.0426558 \\
0.0428329 \\
0.0433166 \\
0.0444422\end{array}$ & $\begin{array}{l}0.0513355 \\
0.0513498 \\
0.0515979 \\
0.0522952\end{array}$ & $\begin{array}{l}0.0644920 \\
0.0645144 \\
0.0649023\end{array}$ & $\begin{array}{l}0.0867166 \\
0.0867573\end{array}$ & 0.1320971 \\
\hline
\end{tabular}

\section{Relative Efficiency}

Suppose we have a sample of size 8 from the Rayleigh distribution

$$
f(x)=\frac{2 x}{\lambda^{2}} e^{-x^{2} / \lambda^{2}} \quad 0 \leq X \leq \infty
$$


The number of observations censored in the left tail $r=2$ and number of observations censored on the right tail is $s=3$ out of sample size 8 . Then using table 3 we find the coefficients $\mathrm{C}_{2}, \mathrm{C}_{3}$ and $\mathrm{C}_{4}$ of $\lambda^{*}$ by Best Linear Unbiased estimate as follows

$$
\begin{aligned}
& \mathrm{C}_{2}=0.239405 \\
& \mathrm{C}_{3}=0.1557252 \\
& \mathrm{C}_{4}=0.8010645
\end{aligned}
$$

$\operatorname{Var}\left(\lambda^{*}\right)=0.0515681 \quad \lambda^{2}$ (also using table 4 we have).

Similarly from table 5 we find the coefficients $g_{2}, g_{3}$ and $g_{4}$ of nearly best linear estimate by Blom's method $\lambda_{1}^{*}$ for doubly censored sample for $r=2$ and $s=3$ for $n=8$ as

$$
\begin{aligned}
& g_{1}=.1975643 \\
& g_{2}=0.1510240 \\
& g_{3}=0.8280674 \text { and (from table 6) we find } \operatorname{Var}\left(\lambda_{1}^{*}\right)=0.051561 . \\
& \text { Relative efficiency }=\frac{0.05115681}{.0515979}=.9999
\end{aligned}
$$

Thus we conclude that Blom's approximate method can replace Lloyd's method which can not be used for large sample and for distributions the exact variance matrix is not available. Hirai (1972) has considered the Quadratic coefficient method as a special case of Downton's (1966) general theory for complete sample. No viable technique is available to extend this method to doubly censored data although attempt has been made for multi-censored data by (Hirai, Ahmad Saeed Akhter and Memon, 2008). We are trying to compare Lloyds' and Blom's method with Gupta (1952) the simplified linear estimates.

\section{References}

1. Adatia (1995). Best Linear Estimator of the Rayleigh Scale Parameter Based on Fairly Large Censored Sample: the Transaction of Reliability, Vol. 44, No.2 pp $302-309$.

2. Blom, G. (1958). Statistical Estimates and Transformed Beta Variables. New York, John Willey \& Sons, Inc.

3. Dyer, D. D., Whisenand, C. W. (1973). Best Linear Unbiased estimator of the parameter of the Rayleigh distribution: Part-II optimum theory for selected order statistics. I.EEE Trans, Vol.60, 1965.

4. Downton, F. (1966). Linear estimator with polynomial coefficients Biometrika, 53 pp $315-327$. 
5. Govindarajulu, Z. and Goshi, M. (1968). Best Linear Unbiased estimation of location and scale parameters using ordered observations, report of statistical research application, Japanese Union of Scientists and Engineers, 15 (2), 1-14.

6. Gupta, A. K. (1952). Estimation of the mean and standard deviation of a normal population from a censored sample biometrika. Vol.39, pp 260-273.

7. Hirai A. S. (1972). Estimation of scale and location parameters using quadratic coefficients. The Australian Journal of Statistics Canberra. Vol.14, No.3, pp 220-223.

8. Hirai A. S. (1976). Estimation of the scale parameter from the type II Rayleigh distribution. Punjab University Journal of Mathematics, pp 61-67.

9. Hirai A. S. (1978). Moments of order statistics from the Rayleigh distribution Journal of Research, Institute of Statistical Research and Training, Bangla Desh. Vol. 12, No.2, pp 1-6, July 1978.

10. Hirai A. S., Akhtar, A. S. and Memon (2008). Estimation of location and scale parameters from multicensored data from the Rayleigh distribution (Accepted for publication). Biannual Research Journal "Grassroots" Pakistan Study Centre, University of Sindh, Jamshoro.

11. Johnson, Kotz and Blakreshanan (1994). Continuous Univariate distributions. Vol. I, $2^{\text {nd }}$ ed., John Wiley \& Sons, Inc. New York.

12. Lloyd, E. H. (1952). Least squares estimation of location and scale parameters using order statistics, Biometrika, 30, pp 88-95.

13. Rayleigh, L. (1880). On the resultant of a large number of vibrations of the some pitch and of arbitrary phase. M.Phil, Mag; 10, pp 73-78.

14. Sarham and Greenberg (1958). Contribution to order Statistics. John Wiley \& Sons, Inc. New York.

15. Weibull (1951). A statistical distribution of wide applicability. J. App. Mach, 18 pp $293-97$. 